\title{
Right ventricular and right atrial function and deformation in patients with subclinical hypothyroidism: a two- and three-dimensional echocardiographic study
}

\author{
Marijana Tadic', Sanja Ilic ${ }^{2}$ and Vera Celic ${ }^{1,3}$ \\ ${ }^{1}$ Cardiology Department and ${ }^{2}$ Department of Endocrinology, University Clinical Hospital Center 'Dr Dragisa \\ Misovic', Heroja Milana Tepica 1, 11000 Belgrade, Serbia and ${ }^{3}$ Faculty of Medicine, Doktora Subotica 6, \\ 11000 Belgrade, Serbia
}

Correspondence

should be addressed to

M Tadic

Email

marijana_tadic@hotmail.com

\begin{abstract}
Background: We sought to investigate right ventricular (RV) function and deformation assessed by three-dimensional echocardiography (3DE) and speckle tracking in patients with subclinical hypothyroidism (SHT), and to evaluate the influence of levothyroxine ( $\left(-\mathrm{T}_{4}\right)$ therapy on $\mathrm{RV}$ remodeling.

Methods: We included 50 untreated women with SHT and 45 healthy control women matched by age. The $\mathrm{LT}_{4}$ therapy was prescribed to all SHT patients who were followed 1 year after euthyroid status was achieved. All study participants underwent laboratory analyses which included thyroid hormone levels, and complete two-dimensional echocardiography (2DE) and 3DE examinations.

Results: 3DE RV end-diastolic volume and ejection fraction were significantly reduced in the SHT patients before therapy in comparison with the healthy controls and treated SHT subjects. RV longitudinal strain, systolic, and early diastolic strain rates (SRs) were significantly decreased, whereas RV late diastolic SR was increased in the SHT patients before therapy when comparing with the controls. $2 \mathrm{DE}$ speckle tracking imaging revealed that $\mathrm{L}-\mathrm{T}_{4}$ substitution therapy significantly improved RV systolic mechanics, whereas RV diastolic deformation was not completely recovered. Right atrial (RA) function and deformation were significantly impacted by SHT. Replacement $L-T_{4}$ treatment improved but did not completely restore RA mechanics in the SHT patients.

Conclusion: RV and RA function and mechanics are significantly affected by SHT. L-T 4 therapy and 1-year maintenance of euthyroid status improved but did not completely recover RV and RA function and deformation in the SHT patients, which implies that right heart remodeling caused by SHT is not reversible in a 1-year period.
\end{abstract}

\section{Introduction}

The cardiovascular system represents one of the most prominent targets of thyroid hormones, which is easily detected in overt hyper- or hypothyroidism (1). The influence of subclinical thyroid dysfunction on the heart and the cardiovascular system is significantly less studied, and pathophysiological mechanisms of this relationship are still unclear (2). Studies showed that subclinical hypothyroidism (SHT) is associated with left ventricular remodeling, especially hypertrophy and diastolic dysfunction $(3,4,5,6,7,8)$. However, the influence of SHT on the right ventricle (RV), as well as the effect of levothyroxine $\left(\mathrm{L}-\mathrm{T}_{4}\right)$ therapy on $\mathrm{RV}$ remodeling, has been investigated in several studies which used only pulsed and tissue Doppler for the estimation of RV function $(9,10,11,12,13,14)$. (c) 2014 European Society of Endocrinology Printed in Great Britain
Published by Bioscientifica Ltd. 
To our knowledge, there is no study which used three-dimensional echocardiography (3DE) and twodimensional (2DE) speckle tracking imaging for assessment of RV function and mechanics in the SHT patients. Additionally, right atrial (RA) mechanics in the SHT patients have not been evaluated so far.

The aim of our investigation was to determine RV and RA function and deformation in the SHT patients, and to evaluate the effect of substitution $\mathrm{L}_{-} \mathrm{T}_{4}$ therapy on $\mathrm{RV}$ and RA remodeling in these patients using $3 \mathrm{DE}$ and $2 \mathrm{DE}$ speckle tracking imaging.

\section{Methodology}

Our study enrolled 50 female patients with untreated SHT and 45 age-matched healthy female volunteers. The study was conducted at the Endocrinology and Cardiology Department, University Clinical Hospital Center 'Dr Dragisa Misovic' in Belgrade, Serbia, between January 2010 and June 2013. The etiology of SHT in all the patients was chronic autoimmune thyroiditis, which was diagnosed by increased circulating antiperoxidase and/or anti-thyroglobulin autoantibodies and diffuse hypoechogenicity by thyroid ultrasound. The inclusion criteria were age ( $\leq 50$ years) and increased serum TSH level with normal levels of free tri-iodothyronine $\left(\mathrm{FT}_{3}\right)$ and free thyroxine $\left(\mathrm{FT}_{4}\right)$. Subjects with symptoms or signs of cardiovascular disease (arterial hypertension, myocardial infarction, atrial fibrillation, heart failure, congenital heart disease, valvular disease), obesity (BMI $\geq 30 \mathrm{~kg} / \mathrm{m}^{2}$ ), asthma, chronic obstructive lung disease, neoplastic disease, cirrhosis of the liver, kidney failure, sleeping disorders, or type 2 diabetes mellitus, as well as professional athletes, were excluded from the study.

Anthropometric measures (height, weight) and laboratory analyses (level of thyroid hormones, total cholesterol, LDL and HDL cholesterol, triglycerides) were taken from all the subjects included in the study. Data about smoking habits, family history of coronary artery disease (CAD), asthma, and obstructive pulmonary disease were taken from all study subjects. Fasting venous blood samples were drawn between 0800 and $0900 \mathrm{~h}$. None of the participants used any medication before their inclusion into the study. Normal ranges for $\mathrm{FT}_{3}, \mathrm{FT}_{4}$, and $\mathrm{TSH}$, were $1.5-4.1 \mathrm{pg} / \mathrm{ml}, \quad 11.5-22.7 \mathrm{pmol} / \mathrm{l}$, and $0.4-4 \mathrm{mIU} / 1$ respectively. $\mathrm{FT}_{3}$ level was determined by IMMULITE 1000, a competitive analog-based immunoassay; $\mathrm{FT}_{4}$ level was assessed by IMMULITE 2000 enzyme-labeled chemiluminescent competitive immunoassay; and TSH level was determined by using IMMULITE 2000, third generation
TSH, two-site chemiluminescent immunometric assay. After the baseline assessment, the patients with SHT were assigned to receive $\mathrm{L}_{-} \mathrm{T}_{4}$ replacement, starting with $25 \mu \mathrm{g} /$ day. TSH was measured every 8 weeks in order to adjust the dose. Euthyroid state was achieved with a mean dose of $74 \mu \mathrm{g} /$ day in $18.2 \pm 5.3$ weeks. Echocardiographic examination was performed before starting the treatment and 1 year after euthyroid state had been achieved by L-T 4 treatment. BMI and body surface area (BSA) were calculated for each patient. The study was approved by the local ethics committee and informed consent was obtained from all the participants.

\section{Echocardiography}

Echocardiographic examination was performed by using a $2.5 \mathrm{MHz}$ transducer with a harmonic capability of a Vivid 7 ultrasound machine (GE Healthcare, Horten, Norway).

\section{Standard 2DE examination}

The values of all 2DE parameters were obtained as the average value of three consecutive cardiac cycles. The LV end-systolic and end-diastolic diameters (LVEDD), the left ventricle posterior wall (PWT), and interventricular septum thickness were determined according to the current recommendations (15). Relative wall thickness was calculated as $(2 \times \mathrm{PWT}) / \mathrm{LVEDD}$. Left ventricular ejection fraction (EF) was estimated by using the biplane method. Left ventricular mass was calculated using the Devereux formula (16) and indexed for height powered to 2.7.

Transmitral Doppler inflow and tissue pulsed Doppler were obtained in the apical four-chamber view. Pulsed Doppler measurements included the transmitral early diastolic peak flow velocity (E), late diastolic flow velocity (A), and their ratio (E/A) (17).

\section{RV and atrium}

The RV internal end-diastolic diameter was measured in M-mode in the parasternal long-axis view (18). RV enddiastolic thickness was measured in the subcostal view (18). The RV fractional area change (RV FAC) was measured from the apical four-chamber view. RV FAC was calculated using the formula: (end-diastolic areaend-systolic area)/end-diastolic area (18). The RA transverse and longitudinal diameters were measured in the apical four-chamber view at the ventricular endsystole (18). RA areas and volumes were obtained by using the biplane method of discs formula (18). RA EF was 
calculated as (RA volume maximum-RA volume minimum)/RA volume maximum.

Tricuspid flow velocities were achieved by the standard pulsed wave Doppler technique in the apical four-chamber view. The following parameters were determined: early diastolic peak flow velocity $\left(E_{t}\right)$, late diastolic flow velocity $\left(A_{t}\right)$, their ratio $(E / A)_{t}$, and $E_{t}$ velocity deceleration time $\left(\mathrm{DT}_{\mathrm{t}}\right)$. Tissue Doppler imaging was used to obtain the RV myocardial velocities in the apical four-chamber view with a sample volume placed at the lateral segment of the tricuspid annulus during early diastole $\left(\mathrm{e}_{\mathrm{t}}^{\prime}\right)$ and systole $\left(\mathrm{s}_{\mathrm{t}}\right)(18)$. $\left(\mathrm{E} / \mathrm{e}^{\prime}\right)_{\mathrm{t}}$ ratio of the RV was determined by using previously estimated Doppler values.

RV global systolic function was assessed as the tricuspid annular plane systolic excursion (TAPSE), which was measured as the difference between the distance among the tricuspid annulus and RV apex at the enddiastole and end-systole of the same cardiac cycle (18).

The parameters necessary for the calculation of the Tei index of the RV were obtained by the tissue Doppler in the apical four-chamber view, according to specific guidelines $(18,19)$.

Assessment of the RV systolic or diastolic dysfunction, along with the global function, was based on the current recommendations (18). RV systolic blood pressure (RVSP) was assessed in a subset of patients with minimal/mild tricuspid regurgitation.

\section{Two-dimensional strain and strain rate}

2DE strain imaging was performed by using three consecutive cardiac cycles of $2 \mathrm{DE}$ images in the apical four-chamber view at the end of expiration (18). The frame rate ranged between 60 and 80 frames/s. Commercially available software EchoPAC 110.1.2, GE Healthcare, was used for the $2 \mathrm{DE}$ strain analysis. The variables which were used for evaluation of systolic function and contractility were the longitudinal peak and systolic strain rate (SR) respectively. Parameters of early myocardial relaxation and late ventricular filling were estimated by early and late diastolic SR. We estimated peak longitudinal strain, systolic and diastolic SRs for the RV, and interventricular septum separately.

The RA speckle tracking analysis was done after the endocardial border was manually traced in the fourchamber view. Six longitudinal strain curves were generated by the software for each atrial segment. RA peak atrial longitudinal strain was calculated by averaging values observed in all RA segments. RA peak systolic SR was measured at RV systolic phase, while early and late RA SRs were measured during early RV filling and throughout late $\mathrm{RV}$ diastolic phase respectively.

\section{DE acquisition}

A full-volume acquisition of the RV required for further analyses was obtained by harmonic imaging from an apical approach. Six electrocardiogram-gated consecutive beats were acquired during end-expiratory breath-hold to generate full volume. All data sets were stored digitally and analyzed by the commercially available software, RV TomTec (EchoPAC 110.1.2, GE Healthcare), which was used for the off-line analysis of RV volumes, stroke volume, and EF. Frame rates were between 20 and 30 frames/s.

\section{Statistical analysis}

Continuous variables were presented as mean \pm s.D. and were compared by using the two-tailed Student's $t$-test as they showed normal distribution. Comparisons between the controls and the patients were performed by an independent-samples $t$-test. The data before and after $\mathrm{L}-\mathrm{T}_{4}$ therapy were compared by a paired-samples $t$-test. The differences in TSH levels presented as median values were analyzed using the Mann-Whitney $U$ test or Wilcoxon test. The differences in proportions were compared by using the $\chi^{2}$-test. The correlations were determined by the Pearson rank correlation test. The $P$ value $<0.05$ was considered statistically significant.

\section{Results}

Basic demographic characteristics and clinical parameters of the study population are presented in Table 1 . There were no differences in the prevalence of smoking, family history of CADs, or obstructive pulmonary disease between the two groups (Table 1). The controls and the SHT patients did not differ in age, BMI, BSA, heart rate, or blood pressure. As it is expected, $\mathrm{FT}_{3}$ and $\mathrm{FT}_{4}$ level were similar between the controls and the SHT participants (before and after substitution therapy), whereas TSH was significantly higher in the SHT patients at baseline in comparison with the controls or values after treatment (Table 1). Triglyceride level and HDL cholesterol were similar between healthy volunteers and the SHT patients. On the other hand, total cholesterol and LDL cholesterol progressively decreased from the SHT patients before therapy, amongst the SHT patients after therapy, to the controls. 
Table 1 Demographic characteristics and clinical parameters of study population.

\begin{tabular}{l}
\hline \\
\hline Age (years) \\
BMI $\left(\mathrm{kg} / \mathrm{m}^{2}\right)$ \\
BSA $\left(\mathrm{m}^{2}\right)$ \\
Smoking (\%) \\
Positive family history of coronary \\
artery disease (\%) \\
Positive family history of asthma \\
or COPD (\%) \\
Heart rate (beats/min) \\
Clinic systolic BP (mmHg) \\
Clinic diastolic BP (mmHg) \\
$\mathrm{FT}_{3}$ (pmol/l) \\
$\mathrm{FT}_{4}$ (pmol/l) \\
TSH (mlU/ml) \\
TSH (median (range)) (mlU/ml) \\
Triglycerides (mmol/l) \\
Total cholesterol (mmol/l) \\
LDL cholesterol (mmol/l) \\
HDL cholesterol (mmol/l)
\end{tabular}

\begin{tabular}{c}
\hline Controls $(n=45)$ \\
\hline $38 \pm 7$ \\
$24.4 \pm 4$ \\
$1.71 \pm 0.18$ \\
$14(31)$ \\
$7(15)$ \\
$3(7)$
\end{tabular}

$71 \pm 12$

$122 \pm 8$

$73 \pm 8$

$2.5 \pm 0.7$

$14.5 \pm 2.5$

$2.23 \pm 0.8^{*}$

$2.1(0.8-3.8) *$

$1.21 \pm 0.68$

$4.58 \pm 0.74^{*}+$

$2.6 \pm 0.59 *,+$

$1.52 \pm 0.27$

\begin{tabular}{c}
\hline Baseline SHT $(n=50)$ \\
\hline $40 \pm 6$ \\
$24.7 \pm 4.6$ \\
$1.73 \pm 0.15$ \\
$14(28)$ \\
$9(18)$
\end{tabular}

SHT after 12 months

$(n=50)$

-
$24.5 \pm 4.4$
$1.70 \pm 0.17$
-
-

4 (8)

$70 \pm 11$
$123 \pm 9$
$72 \pm 9$
$2.47 \pm 0.53$
$14.2 \pm 2.1$
$8.23 \pm 2.11^{*, 5}$
$8.1(5.1-11)^{*, 5}$
$1.33 \pm 0.66$
$5.44 \pm 0.92^{*, \neq}$
$3.3 \pm 0.7^{*, \neq}$
$1.44 \pm 0.24$

$\mathrm{BSA}$, body surface area; $\mathrm{BP}$, blood pressure; COPD, chronic pulmonary disease; $\mathrm{FT}_{3}$, free tri-iodothyronine; $\mathrm{FT}_{4}$, free thyroxine; TSH, thyroid-stimulating hormone; SHT, subclinical hypothyroidism. ${ }^{*} P<0.01$ for controls vs baseline, ${ }^{\dagger} P<0.01$ for controls vs SHT after 12 months, ${ }^{\ddagger} P<0.05$ for baseline vs SHT after 12 months, ${ }^{\S} P<0.01$ for baseline vs SHT after 12 months.

\section{DE left ventricular and atrial parameters}

Left ventricular diameters were similar between the controls and the SHT patients. Interventricular septum thickness and relative wall thickness were increased in the SHT patients at baseline in comparison with the controls (Table 2). The left atrial diameter was similar between the observed groups. The LV mass index was increased in the SHT patients at baseline in comparison with the controls and the SHT patients after therapy. LV EF was similar between the groups. Transmitral E/A ratio was significantly decreased in the SHT patients before therapy compared with the controls and the SHT patients after therapy (Table 2).

\section{DE RV and atrial parameters}

$\mathrm{RV}$ and RA diameters, as well as RV end-systolic and enddiastolic areas, were similar among the groups (Table 2). The RV thickness was increased in the SHT patients at baseline in comparison with the controls and the treated patients. The RA volumes were higher in untreated SHT participants than in the controls. The RA EF was lower in the SHT patients before therapy than in the controls or the treated SHT patients (Table 2).

Transtricuspid $E / A_{t}$ ratio is significantly decreased, and $\mathrm{E} / \mathrm{e}^{\prime}{ }_{\mathrm{t}}$ ratio is significantly increased, in the SHT patients in comparison with controls and the SHT patients after therapy (Table 2). Parameters of the RV systolic function such as TAPSE and $s_{t}$ were similar between the observed groups. However, the Tei index, an indicator of the RV global function, is significantly increased in the SHT patients at baseline compared with healthy controls and the treated participants (Table 2).

\section{DE RV parameters}

The RV end-diastolic and stroke volumes are significantly lower in untreated SHT patients in comparison with the controls and the SHT subjects after $\mathrm{L}_{-} \mathrm{T}_{4}$ therapy (Table 2 ). Interestingly, end-systolic RV volume was similar between the observed subjects. These hemodynamic changes result in lower 3DE RV EF among untreated SHT subjects (Table 2).

\section{RV and atrial function: two-dimensional speckle tracking imaging}

The global RV and interventricular septum longitudinal strain were decreased in the SHT subjects before the substitution therapy as opposed to the controls, but similar with treated SHT patients (Table 3). Systolic RV SR was decreased in untreated SHT patients in comparison with the controls and treated subjects. Early diastolic RV SR was significantly increased, whereas late diastolic RV SR was significantly decreased, in the controls compared with the SHT patients (before and after therapy). 
Table 2 Echocardiographic parameters of left and right ventricular structure and function in the study population.

\begin{tabular}{|c|c|c|c|}
\hline & Controls $(n=45)$ & Baseline SHT $(n=50)$ & $\begin{array}{l}\text { SHT after } 12 \text { months } \\
\qquad(n=50)\end{array}$ \\
\hline \multicolumn{4}{|c|}{ 2D left ventricular and atrial parameters } \\
\hline LVEDD (mm) & $45.2 \pm 4.4$ & $46.1 \pm 4.7$ & $45.5 \pm 4.6$ \\
\hline LVESD (mm) & $31.1 \pm 3.6$ & $32 \pm 4.3$ & $32.1 \pm 4.2$ \\
\hline IVS $(\mathrm{mm})$ & $8.9 \pm 1^{\dagger}$ & $9.4 \pm 1.2^{\dagger}$ & $9.1 \pm 1.1$ \\
\hline RWT & $0.37 \pm 0.04^{\dagger}$ & $0.39 \pm 0.03^{\dagger}$ & $0.38 \pm 0.03$ \\
\hline $\mathrm{LA}(\mathrm{mm})$ & $35 \pm 3.4$ & $35.8 \pm 3.7$ & $35.5 \pm 3.6$ \\
\hline $\mathrm{LVM} / \mathrm{Ht}^{2.7}\left(\mathrm{~g} / \mathrm{m}^{2.7}\right)$ & $39.8 \pm 4.7^{\dagger}$ & $44.3 \pm 5.1^{\dagger, \|}$ & $40.8 \pm 4.9^{\| \prime}$ \\
\hline EF (\%) & $64 \pm 4$ & $63 \pm 5$ & $64 \pm 5$ \\
\hline$(E / A)_{m}$ ratio & $1.48 \pm 0.35^{\dagger}$ & $1.19 \pm 0.39^{\dagger, \|}$ & $1.39 \pm 0.33^{\| \prime}$ \\
\hline \multicolumn{4}{|c|}{ 2DE right ventricular and atrial parameters } \\
\hline RVDd outflow (mm) & $23.5 \pm 2.4$ & $24.2 \pm 2.7$ & $23.8 \pm 2.5$ \\
\hline RVTd subcostal (mm) & $3.9 \pm 0.6 *$ & $4.2 \pm 0.8^{*, \S}$ & $3.8 \pm 0.7^{\S}$ \\
\hline RV EDA $\left(\mathrm{cm}^{2}\right)$ & $15 \pm 3.4$ & $15.5 \pm 3.8$ & $15.2 \pm 3.5$ \\
\hline $\operatorname{RV} \operatorname{ESA}\left(\mathrm{cm}^{2}\right)$ & $7.3 \pm 3$ & $7.8 \pm 3.5$ & $7.5 \pm 3.1$ \\
\hline RV FAC (\%) & $51 \pm 5$ & $50 \pm 4$ & $51 \pm 4$ \\
\hline RA long axis (mm) & $37.9 \pm 6.5$ & $38.8 \pm 6.9$ & $38.2 \pm 6.6$ \\
\hline RA short axis (mm) & $30.7 \pm 4.5$ & $31.4 \pm 4.7$ & $31 \pm 4.1$ \\
\hline RA volume max (ml) & $37 \pm 7$ & $40 \pm 8$ & $38 \pm 6$ \\
\hline RA volume $\min (\mathrm{ml})$ & $14 \pm 4 *$ & $16 \pm 5^{*}$ & $15+5$ \\
\hline RA EF (\%) & $63 \pm 4^{t, \pm}$ & $59 \pm 3^{\dagger, \S}$ & $61 \pm 5^{\ddagger, \S}$ \\
\hline$E / A_{t}$ & $1.45 \pm 0.34^{\dagger}$ & $1.18 \pm 0.27^{\dagger, \|}$ & $1.38 \pm 0.3^{\|}$ \\
\hline$E / e_{t}^{\prime}$ & $4.15 \pm 1.04^{*}$ & $4.76 \pm 1.26^{*, \S}$ & $4.27 \pm 1.11^{\S}$ \\
\hline $\mathrm{s}_{\mathrm{t}}(\mathrm{cm} / \mathrm{s})$ & $14 \pm 2.3$ & $13.6 \pm 2.4$ & $13.8 \pm 2.5$ \\
\hline TAPSE $(\mathrm{mm})$ & $24 \pm 4$ & $23 \pm 3$ & $24 \pm 3$ \\
\hline SPAP $(\mathrm{mmHg})$ & $18 \pm 5$ & $20 \pm 4$ & $19 \pm 4$ \\
\hline RV Tei index & $0.42 \pm 0.05^{\dagger, \neq}$ & $0.5 \pm 0.07^{\dagger, \|}$ & $0.45 \pm 0.06^{\ddagger, \|}$ \\
\hline \multicolumn{4}{|c|}{$3 \mathrm{DE}$ right ventricular parameters } \\
\hline RV EDV $(\mathrm{ml})$ & $121 \pm 19^{\dagger}$ & $106 \pm 16^{\dagger, \S}$ & $116 \pm 20^{\S}$ \\
\hline RV ESV (ml) & $47 \pm 7$ & $45 \pm 6$ & $46 \pm 6$ \\
\hline RV SV (ml) & $74 \pm 8^{\dagger, \ddagger}$ & $61 \pm 6^{t, \|}$ & $70 \pm 7^{ \pm, \|}$ \\
\hline $\mathrm{RV}_{\mathrm{EF}} \mathrm{F}_{3 \mathrm{D}}(\%)$ & $61 \pm 5^{\dagger}$ & $58 \pm 4^{\dagger, \S}$ & $60 \pm 4^{\S}$ \\
\hline
\end{tabular}

A, late diastolic mitral/tricuspid flow (pulse Doppler); E, early diastolic mitral/tricuspid flow (pulse Doppler); $\mathrm{e}^{\prime}$, peak early diastolic relaxation velocity of the lateral segment of tricuspid annulus (tissue Doppler); EDA, end-diastolic area; EDV, enddiastolic volume; EF, ejection fraction; ESA, end-systolic area; ESV, end-systolic volume; FAC, fractional area change; $\mathrm{Ht}$, height; IVS, interventricular septum; LA, left atrium; LV, left ventricle; LVM, left ventricular mass; RA, right atrium; RV, right ventricle; RVD, right ventricular diameter; RVT, right ventricular wall thickness; RWT, relative wall thickness; SPAP, systolic pressure in pulmonary artery; $\mathrm{SV}$, stroke volume. ${ }^{*} P<0.05$ for controls vs baseline $\mathrm{SHT},{ }^{\dagger} P<0.01$ for controls vs baseline, ${ }^{\ddagger} P<0.05$ for controls vs SHT after 12 months, ${ }^{\S} P<0.05$ for baseline vs SHT after 12 months, $P<0.01$ for baseline vs SHT after 12 months.

Interventricular septum SRs differ only between untreated SHT subjects and the controls (Table 3 ).

The global RA strain is decreased in the SHT patients before therapy compared with the controls (Table 3). Similar results are obtained for RA SRs. Namely, systolic and early diastolic SRs are increased, whereas late diastolic SR is decreased, in the controls compared with the SHT patients (before and after therapy). On the other hand, the difference between untreated and treated SHT patients in RA strain and SRs is statistically insignificant.

\section{Correlation and regression analyses}

In the whole study population, which includes the controls and the SHT patients before and after $\mathrm{L}^{-} \mathrm{T}_{4}$ therapy,
TSH level correlated with RV wall thickness $(r=0.38$, $P<0.01), \mathrm{E} / \mathrm{A}_{\mathrm{t}}$ ratio $(r=-0.34, P=0.01), \mathrm{E} / \mathrm{e}_{\mathrm{t}}^{\prime}(r=0.36$, $P<0.01)$, RV Tei index $(r=0.4, P<0.01)$, 3DE RV EF $(r=$ $-0.45, P<0.01)$, RV global longitudinal strain $(r=-0.47$, $P<0.01)$ and RA global longitudinal strain $(r=-0.42$, $P<0.01)$. However, after adjustment for LV mass index and $\mathrm{RV}$ wall thickness, TSH level was associated only with the RV Tei index ( $\beta=-0.32, P=0.02)$, 3DE RV EF $(\beta=-0.38$, $P<0.01)$, and RV longitudinal strain $(\beta=-0.41, P<0.01)$.

\section{Discussion}

Our study revealed several new findings: i) RV function assessed by 3DE is deteriorated in the SHT subjects; ii) $\mathrm{RV}$ mechanics evaluated by $2 \mathrm{DE}$ strain is significantly 
Table 3 Echocardiographic parameters of right ventricular function (2D strain) in the study population.

\begin{tabular}{|c|c|c|c|}
\hline & $\begin{array}{c}\text { Controls } \\
(n=45)\end{array}$ & $\begin{array}{c}\text { Baseline SHT } \\
\quad(n=50)\end{array}$ & $\begin{array}{c}\text { SHT after } 12 \text { months } \\
\qquad(n=50)\end{array}$ \\
\hline \multicolumn{4}{|l|}{ 2DE RV strain and strain rates } \\
\hline \multicolumn{4}{|l|}{ Longitudinal RV strain (\%) } \\
\hline Global RV & $-29 \pm 6^{\dagger}$ & $-26 \pm 5^{\dagger}$ & $-28 \pm 5$ \\
\hline Septum & $-24 \pm 5^{*}$ & $-22 \pm 4 *$ & $-23 \pm 4$ \\
\hline \multicolumn{4}{|l|}{ RV systolic strain rate (per second) } \\
\hline Global RV & $-1.83 \pm 0.44^{\dagger}$ & $-1.52 \pm 0.41^{\dagger, \S}$ & $-1.69 \pm 0.42^{\S}$ \\
\hline Septum & $-1.65 \pm 0.4^{\dagger}$ & $-1.4 \pm 0.31^{\dagger}$ & $-1.53 \pm 0.38$ \\
\hline \multicolumn{4}{|l|}{ RV early diastolic strain rate (per second) } \\
\hline Global RV & $1.9 \pm 0.41^{\dagger, \neq}$ & $1.6 \pm 0.45^{\dagger}$ & $1.72 \pm 0.42^{\ddagger}$ \\
\hline Septum & $1.76 \pm 0.36^{\dagger}$ & $1.5 \pm 0.4^{\dagger}$ & $1.64 \pm 0.38$ \\
\hline \multicolumn{4}{|l|}{ RV late diastolic strain rate (per second) } \\
\hline Global RV & $1.46 \pm 0.33^{\dagger, \neq}$ & $1.72 \pm 0.36^{\dagger}$ & $1.6 \pm 0.42^{\ddagger}$ \\
\hline Septum & $1.41 \pm 0.3^{\dagger}$ & $1.64 \pm 0.35^{\dagger}$ & $1.52 \pm 0.33$ \\
\hline \multicolumn{4}{|l|}{ 2DE RA strain and strain rate } \\
\hline Longitudinal RA strain (\%) & $46 \pm 7^{\dagger}$ & $42 \pm 6^{\dagger}$ & $44 \pm 6$ \\
\hline RA systolic strain rate (per second) & $2.2 \pm 0.6^{*}$ & $1.95 \pm 0.56 *$ & $2.08 \pm 0.62$ \\
\hline RA early diastolic strain rate (per second) & $-2.43 \pm 0.72 *$ & $-2.12 \pm 0.65^{*}$ & $-2.3 \pm 0.66$ \\
\hline RA late diastolic strain rate (per second) & $-2 \pm 0.6 *$ & $-2.28 \pm 0.67 *$ & $-2.08 \pm 0.62$ \\
\hline
\end{tabular}

impaired in the SHT patients; iii) RA mechanics is also changed in SHT; iv) thyroxine therapy significantly improved RV and RA function and mechanics, but right heart remodeling is not completely reversible even after 1 year of euthyroid status.

The relationship between right heart and SHT is intriguing, and despite the fact that several studies showed an association between $\mathrm{RV}$ remodeling and this kind of thyroid dysfunction $(9,10,11,12,13)$, the pathophysiological explanation and mechanisms are still under debate. At the molecular level, this relationship could be illuminated in several ways. First, altered management of intracellular calcium $(20,21)$; second, changed myocardial fiber orientation and capillary blood flow distribution $(22,23)$; third, reduced cardiac oxygen consumption which is associated with increased peripheral resistance, reduced contractility, and decreased efficiency (24); fourth, decreased degradation of myocardial matrix and increased insulin growth factor 1 which might induce cardiac hypertrophy and further RV dysfunction $(25,26)$; and fifth, dyslipidemia, found also in our investigation, could have an influence on cardiac remodeling (27). An important mechanism which should also be mentioned is increased pulmonary vascular resistance and pulmonary hypertension in SHT patients. Namely, studies showed increased prevalence of hypothyroidism in patients with pulmonary hypertension (28), which aroused the suspicion about an autoimmune pathogenetic link between pulmonary hypertension and hypothyroidism (29). Finally, the ventricular interaction could also be a cornerstone of RV remodeling in SHT in two ways: firstly, through the interventricular septum (30), which transduces pressure and volume overload from the left ventricle to RV; and secondly, by transmission of increased left ventricular filling pressure through the pulmonary vascular bed to the RV.

The $2 \mathrm{DE}$ assessment of the RV is very challenging due to complicated RV architecture that makes it insufficiently accessible for 2DE examination. The introduction of 3DE enables the accurate quantitation of RV volumes, function, and mass, and this accuracy can be compared with the results of MRI, which still remains a gold standard for the RV evaluation $(31,32)$. Additionally, 2DE speckle tracking imaging, unlike conventional tissue Doppler imaging, identifies RV and RA myocardial deformation during the whole cardiac cycle, providing information not only about global strain but regional as well, and it also represents a highly reproducible imaging tool which could easily be used in everyday clinical practice (33).

Our study showed that RV diastolic function was impaired in the SHT patients, which agrees with previous investigations $(9,10,11,12,13)$. The impact of SHT on RV systolic function is more controversial. Our findings revealed that RV systolic function estimated by 3DE and 
2DE speckle tracking imaging was significantly deteriorated in the SHT participants, which was not found previously by using conventional echocardiographic tools $(9,10,11,12,13,14)$. However, Ripoli et al. (34) using cardiac MRI showed that SHT individuals had significantly reduced cardiac preload and increased afterload with a consequent decrease in stroke volume and cardiac output, which concurs with our results about the $\mathrm{RV}$. Our results also could be a consequence of increased RV wall thickness among the SHT patients before $\mathrm{L}^{-\mathrm{T}_{4}}$ therapy, which was also found by Kosar et al. (35) in clinical hypothyroid patients.

This study revealed that RV and RA deformation is significantly impacted by SHT, which is a new finding. We would like to emphasize that RV systolic SR which indicates RV systolic deformation was decreased in the SHT patients at baseline in comparison with the controls and treated SHT patients; however, RV early and late diastolic SRs were similar between untreated and treated SHT patients, but significantly deteriorated compared with the controls. These results imply that $\mathrm{L}-\mathrm{T}_{4}$ therapy improved RV systolic and diastolic function, but the improvement is not complete. In other words, RV remodeling in SHT is not completely reversible even after $\mathrm{L}^{-} \mathrm{T}_{4}$ therapy and maintenance of euthyroid status for a year. Other authors showed that replacement therapy resulted in rapid and complete improvement of primarily RV diastolic function $(9,10)$, but we should be aware of the fact that these authors did not use techniques which could detect subtle changes in cardiac function. Our results show that RV systolic function, estimated by $3 \mathrm{DE}$ RV EF, RV global systolic strain, and SRs, was completely restored after L- $\mathrm{T}_{4}$ therapy; whereas RV diastolic function, assessed by RV early and late diastolic SRs, apparently needs more time for improvement. Interestingly, the interventricular septum does not completely follow RV changes in our SHT patients because the difference in deformation exists only between the healthy controls and the SHT patients at baseline, whereas its function after replacement therapy is improved and is not significantly different from the controls. In fact, the difference is between the controls and the SHT patients at baseline. This means that the interventricular septum and left ventricle recover sooner than the RV which is important in determining the duration of treatment in SHT. Furthermore, even after restoration of RV wall thickness in treated SHT patients, $\mathrm{RV}$ mechanics have not been completely recovered, which questions the unfavorable influence of RV hypertrophy on RV function in SHT.
The RA function and deformation in SHT has not been evaluated before. Gaynor et al. (36) previously emphasized the importance of RA three-phasic function: reservoir, conduit, and booster pump function. Our results revealed that systolic and diastolic RA function estimated by 2DE strain are significantly impaired in SHT, which only contributes to the development of RV dysfunction in these patients. This study also showed that 2DE RA EF was reduced in the SHT patients at baseline. Additionally, $\mathrm{L}_{-} \mathrm{T}_{4}$ therapy improved RA function and mechanics, but still not enough to reach the function of the healthy control subjects.

\section{Limitations}

This study has several limitations. Firstly, 3DE estimation of RV structure and function might be significantly influenced by the quality of echocardiographic images, especially during the full-volume acquisition. Secondly, our investigation included only women, which restricts our results to this population. On the other hand, SHT is mostly seen in females, which is why we decided to include only women. Thirdly, the existence of CAD was not excluded by coronary angiography, but we included young females without cardiovascular risk factors, thus expected prevalence of CAD in this population is very low.

\section{Conclusion}

The RV function and deformation assessed by 3DE and 2DE strains are significantly deteriorated in the SHT subjects. Oneyear $\mathrm{L}_{-} \mathrm{T}_{4}$ therapy improved, but did not completely restore RV and RA myocardial function and deformation. This implies that longer substitution therapy is necessary for complete repair of the right heart in SHT patients. Prospective studies are needed to confirm the negative influence of SHT on RV remodeling, as well as to assess the effect of these impairments on morbidity and mortality in this population. Further longitudinal studies are also required to evaluate the effect of $\mathrm{L}-\mathrm{T}_{4}$ replacement therapy on RV remodeling and to define the duration of treatment which is needed for the complete recovery of cardiac function in SHT.

Declaration of interest

The authors declare that there is no conflict of interest that could be perceived as prejudicing the impartiality of the research reported.

\section{Funding}

This research did not receive any specific grant from any funding agency in the public, commercial or not-for-profit sector. 


\section{References}

1 Fazio S, Palmieri EA, Lombardi G \& Biondi B. Effects of thyroid hormone on the cardiovascular system. Recent Progress in Hormone Research 2004 59 31-50. (doi:10.1210/rp.59.1.31)

2 Biondi B, Palmieri EA, Lombardi G \& Fazio S. Effects of subclinical thyroid dysfunction on the heart. Annals of Internal Medicine 2002137 904-914. (doi:10.7326/0003-4819-137-11-200212030-00011)

3 Biondi B, Fazio S, Palmieri EA, Carella C, Panza N, Cittadini A, Bonè F, Lombardi G \& Saccà L. Left ventricular diastolic dysfunction in patients with subclinical hypothyroidism. Journal of Clinical Endocrinology and Metabolism 199984 2064-2067. (doi:10.1210/jc.84.6.2064)

4 Vitale G, Galderisi M, Lupoli GA, Celentano A, Pietropaolo I, Parenti N, De Divitiis O \& Lupoli G. Left ventricular myocardial impairment in subclinical hypothyroidism assessed by a new ultrasound tool: pulsed tissue Doppler. Journal of Clinical Endocrinology and Metabolism 200287 4350-4355. (doi:10.1210/jc.2002-011764)

5 Yazici M, Gorgulu S, Sertbas Y, Erbilen E, Albayrak S, Yildiz O \& Uyan C. Effects of thyroxin therapy on cardiac function in patients with subclinical hypothyroidism: index of myocardial performance in the evaluation of left ventricular function. International Journal of Cardiology 200495 135-143. (doi:10.1016/j.ijcard.2003.05.015)

6 Arinc H, Gunduz H, Tamer A, Seyfeli E, Kanat M, Ozhan H, Akdemir R \& Uyan C. Tissue Doppler echocardiography in evaluation of cardiac effects of subclinical hypothyroidism. International Journal of Cardiovascular Imaging 200622 177-186. (doi:10.1007/s10554-005-9030-2)

7 Martins RM, Fonseca RH, Duarte MM, Reuters VS, Ferreira MM, Almeida C, Buescu A, Teixeira Pde F \& Vaisman M. Impact of subclinical hypothyroidism treatment in systolic and diastolic cardiac function. Arquivos Brasileiros de Endocrinologia e Metabologia 201155 460-467. (doi:10.1590/S0004-27302011000700005)

8 Meena CL, Meena RD, Nawal R, Meena VK, Bharti A \& Meena LP. Assessment of left ventricular diastolic dysfunction in sub-clinical hypothyroidism. Acta Informatica Medica 201220 218-220. (doi:10.5455/aim.2012.20.21-218-220)

9 Turhan S, Tulunay C, Ozduman Cin M, Gursoy A, Kilickap M, Dincer I, Candemir B, Gullu S \& Erol C. Effects of thyroxine therapy on right ventricular systolic and diastolic function in patients with subclinical hypothyroidism: a study by pulsed wave tissue Doppler imaging. Journal of Clinical Endocrinology and Metabolism 200691 3490-3493. (doi:10.1210/jc.2006-0810)

10 Oner FA, Yurdakul S, Oner E, Uzum AK \& Erguney M. Evaluation of the effect of L-thyroxin therapy on cardiac functions by using novel tissue Doppler-derived indices in patients with subclinical hypothyroidism. Acta Cardiologica 201166 47-55.

11 Oner FA, Yurdakul S, Oner E, Arslantaş MK, Usta M \& Ergüney M. Evaluation of ventricular functions using tissue Doppler echocardiography in patients with subclinical hypothyroidism. Türk Kardiyoloji Derneği arşivi 201139 129-136. (doi:10.5543/tkda.2011.01282)

12 Kosar F, Sahin I, Turan N, Topal E, Aksoy Y \& Taskapan C. Evaluation of right and left ventricular function using pulsed-wave tissue Doppler echocardiography in patients with subclinical hypothyroidism. Journal of Endocrinological Investigation 200528 704-710.

13 Arinc H, Gunduz H, Tamer A, Seyfeli E, Kanat M, Ozhan H, Akdemir R, Celebi H \& Uyan C. Evaluation of right ventricular function in patients with thyroid dysfunction. Cardiology 2006105 89-94. (doi:10.1159/ 000089855)

14 Niafar M, Toufan M, Ghafoori S \& Aghamohammadzadeh N. Subclinical hypothyroidism effects on cardiac function. Pakistan Journal of Biological Sciences 200912 1056-1062. (doi:10.3923/pjbs.2009.1056.1062)

15 Lang RM, Bierig M, Devereux RB, Flachskampf FA, Foster E, Pellikka PA, Picard MH, Roman MJ, Seward J, Shanewise J et al. American Society of Echocardiography's Nomenclature and Standards Committee; Task Force on Chamber Quantification; American College of Cardiology Echocardiography Committee; American Heart Association; European Association of Echocardiography. European Society of Cardiology.
Recommendations for chamber quantification. European Journal of Echocardiography 20067 79-108. (doi:10.1016/j.euje.2005.12.014)

16 de Simone G, Daniels SR, Devereux RB, Meyer RA, Roman MJ, de Divitiis $\mathrm{O} \&$ Alderman $\mathrm{MH}$. Left ventricular mass and body size in normotensive children and adults: assessment of allometric relations and impact of overweight. Journal of the American College of Cardiology 199220 1251-1260. (doi:10.1016/0735-1097(92)90385-Z)

17 Quinones MA, Otto CM, Stoddard M, Waggoner A \& Zoghbi WA. Recommendations for quantification of Doppler echocardiography: a report from the Doppler quantification task force of the nomenclature and standards committee of the American Society of Echocardiography. Journal of the American Society of Echocardiography 200215 167-184. (doi:10.1067/mje.2002.120202)

18 Rudski LG, Lai WW, Afilalo J, Hua L, Handschumacher MD, Chandrasekaran K, Solomon SD, Louie EK \& Schiller NB. Guidelines for the echocardiographic assessment of the right heart in adults: a report from the American Society of Echocardiography endorsed by the European Association of Echocardiography, a registered branch of the European Society of Cardiology, and the Canadian Society of Echocardiography. Journal of the American Society of Echocardiography 201023 685-713. (doi:10.1016/j.echo.2010.05.010)

19 Tei C, Ling LH, Hodge DO, Bailey KR, Oh JK, Rodeheffer RJ, Tajik AJ \& Seward JB. New index of combined systolic and diastolic myocardial performance: a simple and reproducible measure of cardiac function - a study in normals and dilated cardiomyopathy. Journal of Cardiology 199526 357-366.

20 Dillmann WH. Biochemical basis of thyroid hormone action in the heart. American Journal of Medicine 199088 626-632. (doi:10.1016/ 0002-9343(90)90530-Q)

21 Ojamaa $\mathrm{K} \&$ Klein I. In vivo regulation of recombinant cardiac myosin heavy chain gene expression by thyroid hormone. Endocrinology 1993 132 1002-1010. (doi:10.1210/en.132.3.1002)

22 Brenta G, Mutti LA, Schnitman M, Fretes O, Perrone A \& Matute ML. Assessment of left ventricular diastolic function by radionuclide ventriculography at rest and exercise in subclinical hypothyroidism, and its response to L-thyroxine therapy. American Journal of Cardiology 200391 1327-1330. (doi:10.1016/S0002-9149(03)00322-9)

23 Knapp M, Lisowska A, Sobkowicz B, Tycińska A, Sawicki R \& Musiał W. Myocardial perfusion and intima-media thickness in patients with subclinical hypothyroidism. Advances in Medical Sciences 201358 44-49.

24 Bengel FM, Nekolla SG, Ibrahim T, Weniger C, Ziegler SI \& Schwaiger M. Effect of thyroid hormones on cardiac function, geometry, and oxidative metabolism assessed noninvasively by positron emission tomography and magnetic resonance imaging. Journal of Clinical Endocrinology and Metabolism 200085 1822-1827. (doi:10.1210/jc.85.5.1822)

25 Ghose Roy S, Mishra S, Ghosh G \& Bandyopadhyay A. Thyroid hormone induces myocardial matrix degradation by activating matrix metalloproteinase-1. Matrix Biology 200726 269-279. (doi:10.1016/j. matbio.2006.12.005)

26 Sabatino L, Gliozheni E, Molinaro S, Bonotti A, Azzolina S, Popoff G, Carpi A \& Iervasi G. Thyroid hormone receptor and IGF1/IGFR systems: possible relations in the human heart. Biomedicine \& Pharmacotherapy 200761 457-462. (doi:10.1016/j.biopha.2007.04.002)

27 Dalen H, Thorstensen A, Romundstad PR, Aase SA, Stoylen A \& Vatten LJ. Cardiovascular risk factors and systolic and diastolic cardiac function: a tissue Doppler and speckle tracking echocardiographic study. Journal of the American Society of Echocardiography 201124 322-332. (doi:10.1016/j.echo.2010.12.010)

28 Curnock AL, Dweik RA, Higgins BH, Saadi HF \& Arroliga AC. High prevalence of hypothyroidism in patients with primary pulmonary hypertension. American Journal of the Medical Sciences 1999318 289-292. (doi:10.1097/00000441-199911000-00001)

29 Badesch DB, Wynne KM, Bonvallet S, Voelkel NF, Ridgway C \& Groves BM. Hypothyroidism and primary pulmonary hypertension: an 
autoimmune pathogenetic link? Annals of Internal Medicine 1993119 44-46. (doi:10.7326/0003-4819-119-1-199307010-00008)

30 Buckberg GD \& RESTORE Group. The ventricular septum: the lion of right ventricular function, and its impact on right ventricular restoration. European Journal of Cardio-Thoracic Surgery 200629 S272-S278. (doi:10.1016/j.ejcts.2006.02.011)

31 Shiota T. 3D echocardiography: evaluation of the right ventricle. Current Opinion in Cardiology 200924 410-414. (doi:10.1097/HCO. 0b013e32832cf8f6)

32 Mor-Avi V \& Lang RM. Three-dimensional echocardiographic evaluation of the heart chambers: size, function, and mass. Cardiology Clinics 200725 241-251. (doi:10.1016/j.ccl.2007.05.003)

33 La Gerche A, Jurcut R \& Voigt JU. Right ventricular function by strain echocardiography. Current Opinion in Cardiology 201025 430-436. (doi:10.1097/HCO.0b013e32833b5f94)
34 Ripoli A, Pingitore A, Favilli B, Bottoni A, Turchi S, Osman NF, De Marchi D, Lombardi M, L'Abbate A \& Iervasi G. Does subclinical hypothyroidism affect cardiac pump performance? Evidence from a magnetic resonance imaging study. Journal of the American College of Cardiology 200545 439-445. (doi:10.1016/j.jacc.2004.10.044)

35 Kosar F, Sahin I, Aksoy Y, Uzer E \& Turan N. Usefulness of pulsed-wave tissue Doppler echocardiography for the assessment of the left and right ventricular function in patients with clinical hypothyroidism. Echocardiography 200623 471-477. (doi:10.1111/j.1540-8175.2006. 00243.x)

36 Gaynor SL, Maniar HS, Prasad SM, Steendijk P \& Moon MR. Reservoir and conduit function of right atrium: impact on right ventricular filling and cardiac output. American Journal of Physiology. Heart and Circulatory Physiology 2005288 H2140-H2145. (doi:10.1152/ajpheart. 00566.2004)

Received 18 August 2013

Revised version received 30 September 2013

Accepted 10 October 2013 